\title{
Spatially Restricted Increase in Polysialic Acid Enhances Corticospinal Axon Branching Related to Target Recognition and Innervation
}

\author{
Maryellen M. Daston, ${ }^{1}$ Martin Bastmeyer, ${ }^{1}$ Urs Rutishauser, ${ }^{2}$ and Dennis D. M. O'Leary ${ }^{1}$ \\ ${ }^{1}$ Molecular Neurobiology Laboratory, The Salk Institute, La Jolla, California 92037, and 'Department of Genetics and \\ Neurosciences, Case Western Reserve University, Cleveland, Ohio 44106
}

\begin{abstract}
The polysialic acid (PSA) modification of the neural cell adhesion molecule (NCAM) has been shown to alter the responses of developing axons to their environment. We have studied the potential role of PSA in regulating the innervation of the spinal cord by corticospinal axons, which occurs by a delayed formation of collateral branches from the parent axons. Developmental changes in the distribution of PSA were examined immunohistochemically using light and electron microscopy. Whereas NCAM is distributed along the entire pathway of rat corticospinal axons as they grow from the cortex to the spinal cord, PSA-modified NCAM does not become evident until later. When PSA becomes evident, it is restricted to the distal segment of these axons from the caudal hindbrain through the spinal cord. The increase in PSA on corticospinal axons coincides with the time that they begin to form collateral branches
\end{abstract}

in the spinal cord. This unique spatiotemporal distribution of PSA suggests its involvement in corticospinal axon branching. To test this hypothesis, PSA was selectively removed by an in vivo injection of endoneuraminidase $\mathrm{N}$. This treatment did not seem to interfere with the pathfinding of corticospinal axons; however, PSA removal delayed the onset of collateral branching by corticospinal axons within the spinal cord and later diminished the magnitude of branching. These findings indicate a role for PSA in the regulation of interstitial axon branching, a crucial step in the process of target recognition and innervation by corticospinal axons.

Key words: axon branching; axon collaterals; axon pathfinding; axon targeting; basilar pons; cell-cell interactions; corticospinal tract; endoneuraminidase $\mathrm{N}$; immunoelectron microscopy; neural cell adhesion molecules; spinal cord
Layer 5 cortical neurons innervate their targets in the midbrain, hindbrain, and spinal cord by the process of "interstitial axon branching” (O'Leary and Terashima, 1988; O'Leary et al., 1990; Kuang and Kalil, 1994; Bastmeyer and O'Leary, 1996). Instead of elaborating an axon that grows toward and into a target, layer 5 cortical neurons extend axons that grow past their targets and remain within their axon tract. Subsequently, collateral branches arise de novo along these axons well behind their advancing growth cones, exit the tract, and grow into their target (Bastmeyer and O'Leary, 1996). The importance of interstitial axon branching as a mode of target recognition and innervation is becoming increasingly apparent as more examples are characterized, including the development of the axonal projection from the hippocampal formation to the mammillary bodies (Stanfield et al., 1987), the retina to the lateral geniculate nucleus (Bhide and Frost, 1991) and tectum (Nakamura and O'Leary, 1989), and the dorsal root ganglia to the spinal cord (Snider et al., 1992).

The ability of an axon to respond to signals that promote the formation and directed extension of collateral branches could be regulated by factors that modify axon-axon or axon-substrate

\footnotetext{
Received March 20, 1996; revised May 22, 1996; accepted June 12, 1996.

This work was supported by National Institutes of Health Grants P01 NS31249 (D.D.M.O.), R01 HD18369 (U.R.), and F32 NS09444-02 (M.M.D.), and Deutsche Forschungsgemeinschaft Fellowship Ba 1034/4 (M.B.). We thank Patrick Burrola (The Salk Institute) for expert technical assistance with the immunoelectron microscopy.

Correspondence should be addressed to Dennis D. M. O'Leary, MNL-O, The Salk Institute, 10010 North Torrey Pines Road, La Jolla, CA 92037.

Dr. Bastmeyer's present address: Universität Konstanz, Fakultät für Biologie, Postfach 5560, D-78434 Konstanz, Germany.

Copyright (C) 1996 Society for Neuroscience $0270-6474 / 96 / 165488-10 \$ 05.00 / 0$
}

interactions. One of the most abundant axonal adhesion molecules, the neural cell adhesion molecule (NCAM) can be modified by the addition of polysialic acid (PSA). The degree of polysialation of NCAM is regulated developmentally (Hoffman et al., 1982; Rothbard et al., 1982), and in general, increases in PSA correspond to periods of axon growth and pathfinding (Rougon et al., 1982; Chuong and Edelman, 1984; Landmesser et al., 1990; Tang et al., 1992). PSA on NCAM has been proposed to attenuate cell-cell or cell-substrate interactions mediated by NCAM and by other cell-surface molecules such as L1 (Rutishauser, 1989). A role for PSA in axon pathfinding and target innervation has been indicated by studies in chick, which show that the enzymatic removal of PSA from growing motor axons interferes with their sorting in the limb plexus and the formation of appropriately patterned peripheral nerves to target muscles (Landmesser et al., 1990; Tang et al., 1992, 1994).

Joosten (1994) recently described a developmental increase in PSA in the corticospinal tract (CST) within the spinal cord, leading him to suggest that PSA may facilitate the branching of corticospinal axons, an issue that we examine here. First, we extended his observations by examining the distribution of PSA along the entire pathway of developing corticospinal axons from the forebrain to the spinal cord. We have found using immunohistochemistry and immunoelectron microscopy that PSA is localized to the distal segment of corticospinal axons and that PSA becomes prominent after corticospinal axons have essentially completed their phase of pathfinding but coincident with the onset of collateral branching. To test whether PSA is involved in the innervation of the spinal cord, we used the specific enzyme endoneuraminidase $\mathrm{N}$ to selectively remove PSA in vivo and 
examined the formation of collateral branches by corticospinal axons in the absence of PSA.

\section{MATERIALS AND METHODS}

\section{Animals}

Rat pups were obtained from timed-pregnant Sprague-Dawley rats purchased from Harlan Sprague-Dawley (Indianapolis, IN). The time of birth was monitored closely. The first $24 \mathrm{hr}$ after birth was considered postnatal day $0(\mathrm{P} 0)$. Rat pups were anesthetized by hypothermia before all procedures. Embryonic day 20 (E20) fetuses were removed by cesarian section from a pregnant rat deeply anesthetized with Nembutal.

\section{Immunohistochemistry}

Light microscopy. Two primary antibodies were used to analyze the distributions of NCAM and PSA: the mouse monoclonal antibody (mAb) 5A5, which recognizes the PSA moiety (Dodd et al., 1988; Acheson et al., 1991), and a rabbit polyclonal, R050, which was made against affinitypurified NCAM derived from mouse brain and recognizes all isoforms of rat NCAM.

Rat pups were perfused through the left ventricle with $4 \%$ paraformaldehyde in $0.1 \mathrm{~m}$ phosphate buffer (PB), $\mathrm{pH}$ 7.4. After perfusion, brains and spinal cords were dissected and placed into the same fixative overnight at $4^{\circ} \mathrm{C}$. Tissue was then cryoprotected by equilibration in $20 \%$ sucrose in PB; $10-12 \mu \mathrm{m}$ cryostat sections were cut and dried onto gelatin-subbed slides. Sections were treated for $30 \mathrm{~min}$ with blocking buffer (PBS with 10\% normal goat serum) followed by overnight incubation at $4^{\circ} \mathrm{C}$ with primary antibodies diluted in blocking buffer. Sections were washed with PBS, incubated for $1 \mathrm{hr}$ at room temperature in the appropriate fluorescent-conjugated secondary antibodies (Jackson ImmunoResearch, West Grove, PA), which were also diluted in blocking buffer, and then mounted in Mowiol with $n$-propyl gallate added to prevent fading.

Electron microscopy. P6 rat pups were perfused with $4 \%$ paraformaldehyde $/ 0.2 \%$ glutaraldehyde in 0.1 м Sörensen's phosphate buffer (SPB), $\mathrm{pH}$ 7.4. The spinal cords were dissected, postfixed for $1 \mathrm{hr}$ in the same fixative at $4{ }^{\circ} \mathrm{C}$, and cut into $200-\mu \mathrm{m}$-thick sections on a vibratome. The sections were freeze-thawed by lowering them into liquid nitrogen for 2-5 sec, treated with $10 \%$ normal goat serum in SPB for $1 \mathrm{hr}$, and incubated overnight at room temperature with mAb 5A5, or for controls, with SPB alone. After three washes in SPB, sections were processed for peroxidase histochemistry using the ABC Elite kit (Vector, Burlingame, CA) essentially as described by Liu et al. (1993), except that SPB was used in all steps. Sections were developed with $0.5 \% \mathrm{DAB}, 0.1 \% \mathrm{H}_{2} \mathrm{O}_{2}$ in SPB for 5-10 min. After three washes in SPB, they were postfixed in 3\% glutaraldehyde in $\mathrm{SPB}$ for $30 \mathrm{~min}$ and in $1 \% \mathrm{OsO}_{4}, 1.5 \%$ potassium ferrocyanide in SPB for $2 \mathrm{hr}$. Sections were dehydrated in a graded series of ethanol and embedded in Durcupan resin (Fluka, Buchs, Switzerland). Ultrathin sections $(70 \mathrm{~nm})$ were cut, poststained with Reynolds lead citrate, and viewed and photographed with a JEOL transmission electron microscope.

\section{In vivo injection of endoneuraminidase $N$ (endo $N$ )}

The endo $\mathrm{N}$ used in this study was purified from bacteriophage (Hallenbeck et al., 1987). This preparation does not exhibit toxicity for cells in culture or in vivo and does not contain detectable amounts of protease activity. It acts optimally at $\mathrm{pH} 7$, does not require cofactors, and specifically cleaves $-2,8$-linked sialic acid polymers with a minimum chain length of eight (Vimr et al., 1984; Hallenbeck et al., 1987). The enzyme is specific for PSA and has no activity toward other sialic acidcontaining components. When injected in vivo in vertebrate embryos, endo $\mathrm{N}$ has been shown to completely remove PSA over wide expanses of developing tissue (Landmesser et al., 1990; Tang et al., 1992, 1994).

Endo $\mathrm{N}$ was injected into the fourth ventricle of P0 rats, within $2-5 \mathrm{hr}$ of birth. To gain access to the fourth ventricle, an incision was made in the skin at the back of the skull, and the neck muscles were reflected to expose the occipitoatlantal ligament. A glass micropipette pulled to a fine tip containing endo $\mathrm{N}$ (or heat-inactivated endo $\mathrm{N}$ ) was used to puncture the ligament. One microliter of enzyme solution was then introduced into the fourth ventricle through the micropipette using a picospritzer (General Valve, Fairfield, NJ). For sham injections, the same surgery was performed, and the ligament was punctured using an empty micropipette. After injections the wound was sutured, and rat pups were returned to their mothers after recovery from hypothermia anesthesia.

\section{Anterograde labeling of CST axons}

1,1'-Dioctodecyl-3,3,3',3'-tetramethylindocarbocyanine perchlorate (DiI) labeling. To label corticospinal axons fluorescently, endo $\mathrm{N}$-treated rat pups and control littermates received bilateral injections of $10 \%$ DiI (Molecular Probes, Eugene, OR) (Honig and Hume, 1989) in dimethyl formamide in sensorimotor cortex $2 \mathrm{~d}$ before they were killed (P2, P3, or P4). Rat pups were perfused at P4, P5, or P6 (all pups of a given litter were perfused at the same age) with $10 \%$ neutral-buffered formalin. Brains and spinal cords were dissected and placed in the same fixative and stored at $4^{\circ} \mathrm{C}$ for $24 \mathrm{hr}$ or longer. Spinal cords were flattened in the dorsoventral plane between two glass slides with spacers of the same thickness as the spinal cords before postfixation to eliminate the natural curvature of the spinal cord. Flattening the spinal cords made it possible to obtain horizontal sections in which the CST did not pass in and out of the plane of section. The rostral halves of the spinal cords were cut in 100 $\mu \mathrm{m}$ horizontal sections using a vibratome. DiI-labeled CST axons were examined and photographed using rhodamine optics.

Biocytin labeling. Rat pups that had been injected with endo $\mathrm{N}$ (or control injections) on P0 received cortical biocytin injections on P4 18-20 hr before perfusion on P5. Injections of 5\% biocytin (Sigma, St. Louis, $\mathrm{MO}$ ) in $0.9 \% \mathrm{NaCl}$ were made in sensorimotor cortex. In each hemisphere, three injections were made along the coronal suture. After perfusion, brains and spinal cords were prepared as described above for DiI-injected animals. Brains and spinal cords were cryoprotected and frozen (as for immunohistochemistry), and 50- $\mu \mathrm{m}$-thick horizontal serial sections of the rostral half of the spinal cords were cut using a freezing
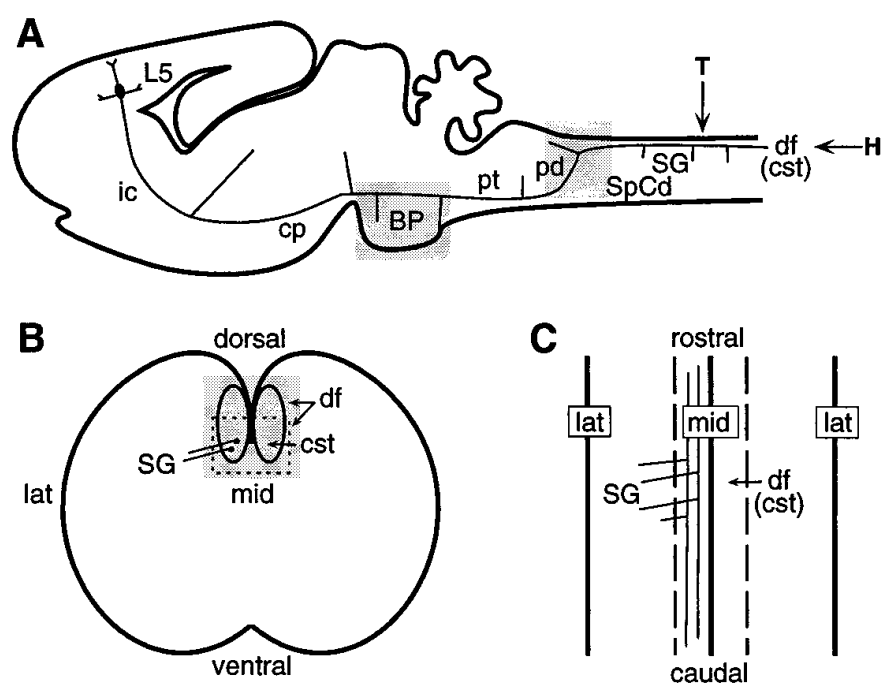

Figure 1. Drawings depicting the CST and anatomical structures relevant to this study. $A$, Sagittal view of an early postnatal rat brain illustrating the pathway of corticospinal axons, which originate from cortical layer 5 (L5) neurons, and the location of collateral branches related to some of their major subcortical targets. The locations and planes of section of subsequent figures are indicated. The shaded boxes surrounding the basilar pons $(B P)$ and the pyramidal decussation $(p d)$ indicate the location of the sagittal sections shown in Figure $2 A-D$ and $E, F$, respectively. The arrow marked $T$ approximates the location of the transverse sections shown in Figures 3 and 6 . The arrow marked $H$ approximates the location of the horizontal sections shown in Figure 7. $B$, Schematic of a transverse section through the spinal cord (marked by the arrow $T$ in $A$ ). The lines extending from the two black dots depict branches (lines) extending laterally into the spinal gray $(S G)$ from parent corticospinal axons (black dots) located in the corticospinal tract (cst), which is in the ventral part of the dorsal funiculus $(d f)$. The dashed box and the shaded box indicate the approximate locations of the panels illustrated in Figures 3 and 6, respectively. $C$, Schematic of a horizontal section through the spinal cord (marked by the arrow $H$ in $A$ ) as in Figure 7. Depicted are four branches extending laterally into the spinal gray from two parent corticospinal axons coursing rostrocaudally through the left CST. Abbreviations: $B P$, basilar pons; $c p$, cerebral peduncle; cst, corticospinal tract; $d f$, dorsal funiculus; ic, internal capsule; lat, lateral; L5, layer 5; mid, midline; $p d$, pyramidal decussation; $p t$, pyramidal tract; $S G$, spinal gray; $S p C d$, spinal cord. 

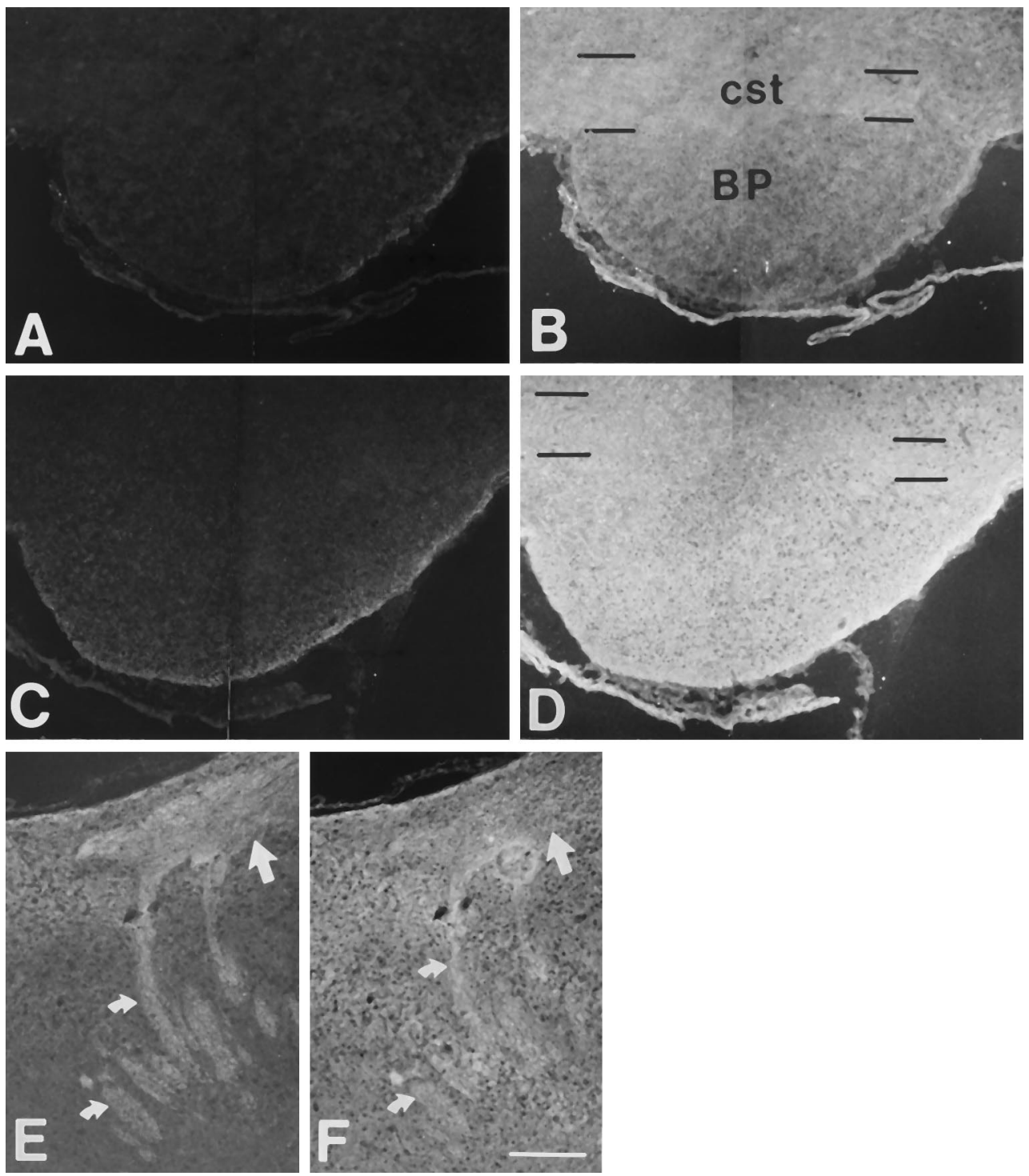

microtome. The sections were mounted and dried onto gelatin-subbed slides. To visualize biocytin-labeled axons, peroxidase histochemistry using the ABC Elite kit (Vector) was carried out essentially as described in Liu et al. (1993), except that tissue sections were mounted on slides before processing and PBS was used in all steps. In addition, a biotinyl tyramine amplification step (Adams, 1992) was added.

\section{Quantification of branching}

For each spinal cord a complete series of sections containing the entire CST was examined. The number of labeled axons was determined by counting every axon that intersected a transverse line $600 \mu \mathrm{m}$ caudal to the hindbrain/spinal cord boundary. The number of branches was determined by counting all branch points along labeled CST axons within the rostral $6 \mathrm{~mm}$ of the spinal cord.

\section{RESULTS}

\section{Distribution of PSA in the developing CST}

Immunohistochemistry was used to determine the temporal and spatial pattern of PSA distribution in the developing corticospinal pathway. The mAb 5A5, which recognizes the PSA moiety, and the polyclonal antibody $\mathrm{R} 050$, which recognizes all isoforms of rat NCAM, were used to double-immunolabel sections of late embryonic and early postnatal rat brains and spinal cords, the period during which corticospinal axons grow through their subcortical
Figure 2. PSA is present caudally but not rostrally in the developing CST. Sagittal sections of rat brains double-immunostained for PSA $(A, C, E)$ and NCAM $(B, D, F)$. $A-D$, PSA was not detectable in the corticospinal tract (cst) (marked by black lines) overlying the basilar pons $(B P)$ at $\mathrm{P} 0(A)$ or $\mathrm{P} 4(C)$, or at E20 (not shown). NCAM was abundant in the tract and in the surrounding area at each of these ages $(B, \mathrm{P} 0 ; D, \mathrm{P} 4)$. $E$, $F$, At P4, PSA was present in the CST more caudally in the hindbrain, where PSA staining highlights the pyramidal decussation (curved arrows) at the hindbrain-spinal cord junction $(E)$. PSA staining is also apparent in the dorsal funiculus of the spinal cord (straight arrow). NCAM was distributed throughout the hindbrain and spinal cord $(F)$. For each antibody, the same exposure times were used for photographing and printing. Rostral is to the left, and dorsal is at the top. The approximate location and orientation of the panels is illustrated in Figure $1 A$. Scale bar (shown in $F$ ): $200 \mu \mathrm{m}$. pathway and extend collateral branches to their brainstem and spinal targets (Stanfield, 1992; O'Leary and Koester, 1993). The pathway of layer 5 corticospinal axons from the cortex to the spinal cord is schematically illustrated in Figure 1.

At E20, the leading corticospinal axons have reached the level of the basilar pons (O'Leary and Terashima, 1988). At this age, however, staining for PSA was detected only in the internal capsule, where corticospinal axons commingle with thalamocortical axons. PSA in the internal capsule may be associated with thalamocortical axons rather than corticospinal axons, because it is not present at more distal positions within the forebrain where the corticospinal pathway becomes distinct (not shown). Even at later ages, PSA staining was not observed over the portion of the corticospinal pathway through the brainstem. For example, PSA staining was absent in the corticospinal pathway over the basilar pons at P0 (Fig. $2 A$ ), when corticospinal axons begin to extend collateral branches to the pons, and at P4 (Fig. $2 C$ ), when branching to the basilar pons is essentially complete. Staining for NCAM, however, was present throughout the brainstem corticospinal pathway at all ages examined (Fig. $2 B, D$ ).

In contrast to the lack of PSA staining within the corticospinal 

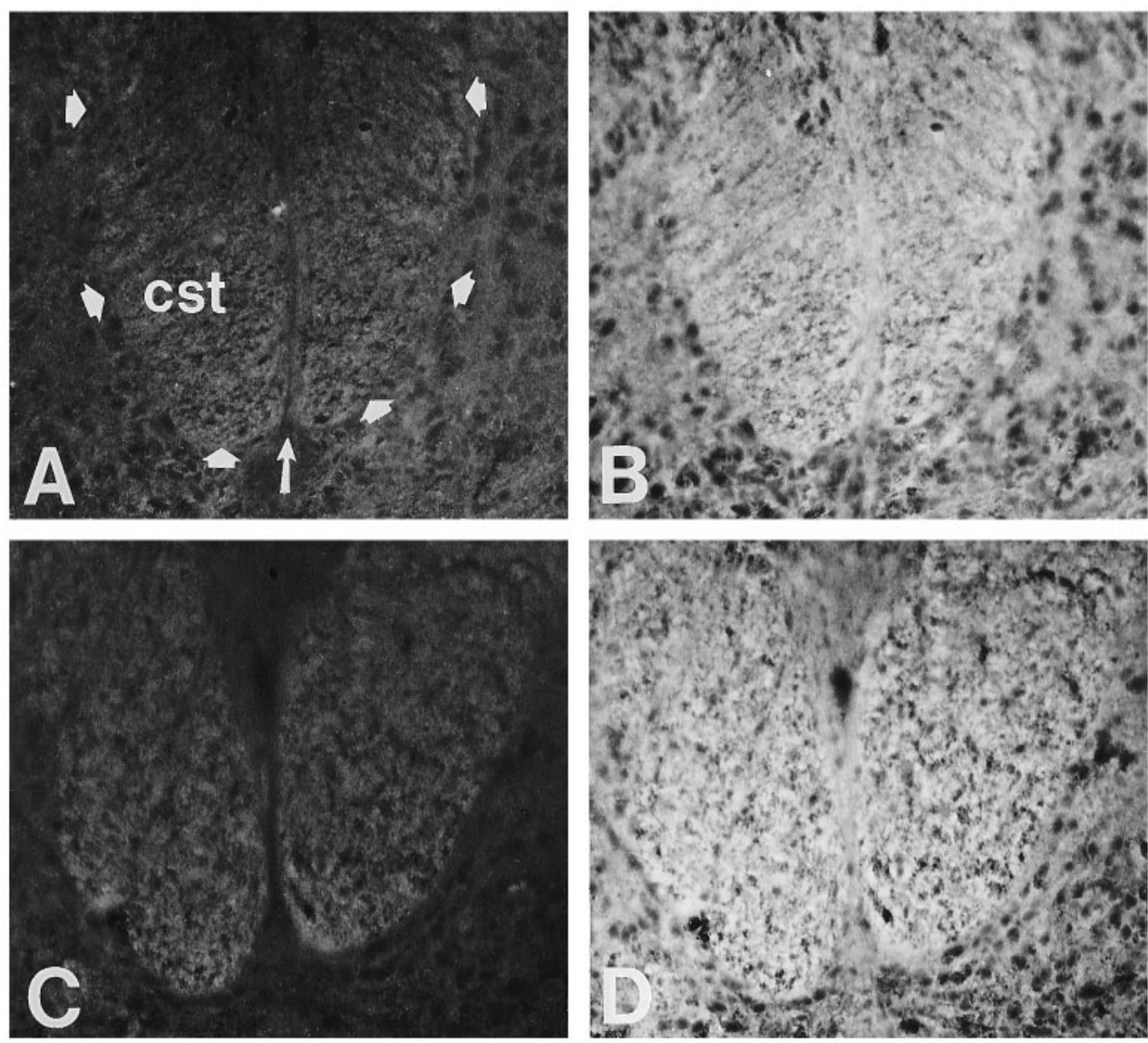

Figure 3. In the spinal cord, PSA increases in the corticospinal tract (cst) at the time of corticospinal axon branching. Transverse sections of rat cervical spinal cords double-immunostained for PSA ( $A$, $C, E)$ and $\operatorname{NCAM}(B, D, F)$. PSA staining is weak in the CST (outlined by thick arrows in $A$; thin arrow marks midline) and spinal gray at $\mathrm{P} 0(A)$ and $\mathrm{P} 2(C)$. At $\mathrm{P} 4$, PSA staining is intense in the CST but remains weak in the surrounding spinal gray $(E)$. NCAM is abundant in the CST and the surrounding spinal gray at $\mathrm{P} 0(B)$, $\mathrm{P} 2(D)$, and $\mathrm{P} 4(F)$. The approximate location and orientation of the panels are illustrated in Figure 1, $A$ (arrow marked $T$ ) and $B$ (dashed box). Dorsal is to the top. Scale bar, $100 \mu \mathrm{m}$.
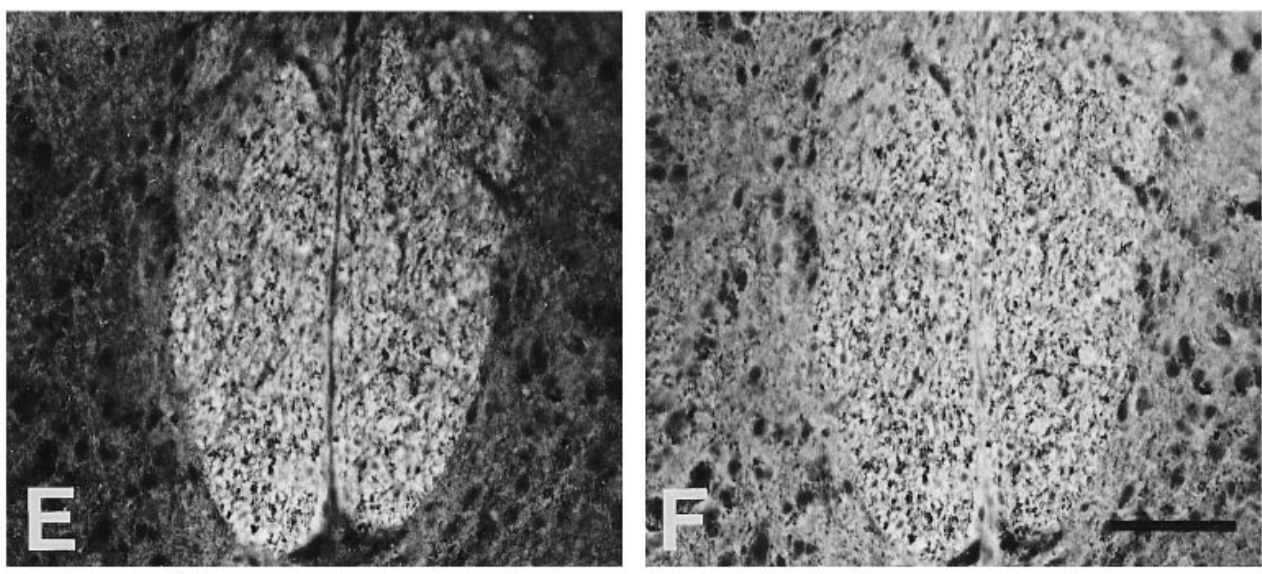

pathway over the basilar pons, in the spinal cord PSA staining becomes prominent specifically within the CST at the onset of axon collateralization. In the rat spinal cord, the CST is a midline structure located in the ventral-most portion of the dorsal funiculus. Corticospinal axons enter the spinal cord before birth, and on P0 have already extended beyond the cervical level of the spinal cord (Schreyer and Jones, 1982). At both P0 (Fig. 3A) and P2 (Fig. 3C), PSA staining was weak in the cervical CST and in the surrounding spinal gray. At P4, however, when corticospinal axons begin to extend collateral branches to the spinal gray (LopezMascaraque and O'Leary, 1991), PSA staining was intense in the cervical CST but remained weak in the surrounding spinal gray (Fig. 3E). Within the dorsal funiculus, the staining specifically increased in the ventrally located CST and was low or absent in its dorsal components, which contain ascending sensory axons (see
Fig. 6A). At all of these ages, NCAM was abundant throughout the dorsal funiculus as well as in the surrounding spinal gray (Fig. 3B,D,F).

The rostral-most location of detectable PSA staining within the corticospinal pathway was the caudal hindbrain. At P4, when there was no detectable PSA staining in the corticospinal pathway in rostral hindbrain or over the basilar pons (Fig. 2C), PSA staining in the caudal hindbrain highlighted the pyramidal decussation (Fig. 2E), although the staining appeared to be less intense than that in the spinal cord. Again, this spatial difference in PSA distribution does not reflect a difference in the distribution of NCAM, which was present throughout the hindbrain (Fig. $2 F$ ).

To determine whether this unique spatiotemporal distribution of PSA in the corticospinal pathway is attributable to a differential distribution of PSA on corticospinal axons or on other cell types 

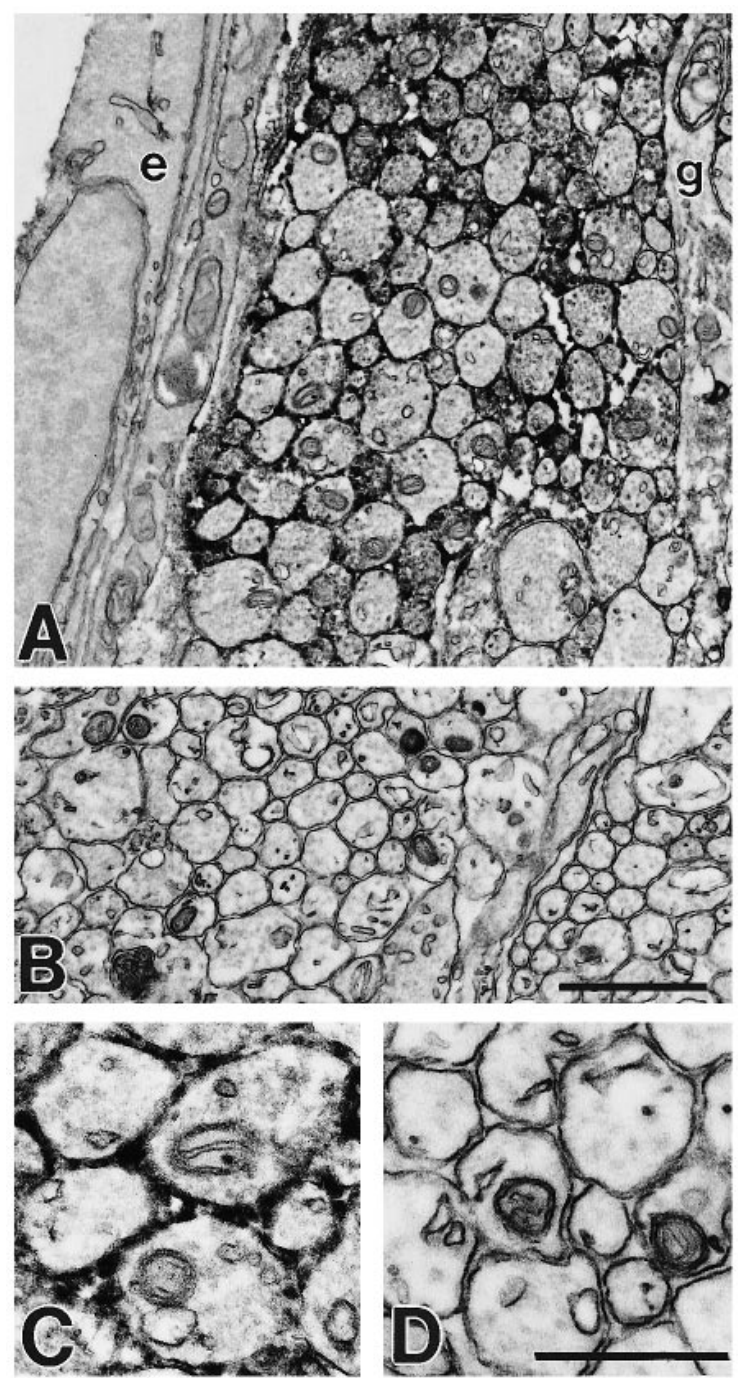

Figure 4. PSA is selectively localized to axonal surfaces in the CST. Pre-embedding immunoelectron microscopy with an anti-PSA mAb and HRP-coupled secondary antibodies. $A, C$, Transverse section of P6 rat cervical spinal cord through the CST demonstrates that PSA immunoreactivity (black reaction product in intercellular spaces between the membranes of apposing axons) is associated with axonal profiles. PSA immunoreactivity is not associated with endothelial $(e)$ or glial $(g)$ cell processes except where they are adjacent to axons. $B, D$, Control section treated in the same way but without primary antibody. Scale bars: $A, B, 1 \mu \mathrm{m} ; C, D$, $0.25 \mu \mathrm{m}$.

in the tract, we used immunoelectron microscopy for subcellular PSA localization. Figure 4 shows electron micrographs of the CST of a P6 rat immunostained with mAb 5A5. Immunoreactivity was localized to the extracellular surface of axonal membranes. PSA immunoreactivity was not associated with the surfaces of other cellular components of the tract, which include immature glia and endothelial cells, except where they were apposed to axons.

These findings on the distribution of PSA-modified NCAM, summarized in Figure 5, indicate that PSA is distributed selectively to the distal portion of corticospinal axons, and rather than being present on the growing axons, PSA increases substantially at the time that corticospinal axons begin to form collateral branches to the spinal gray.

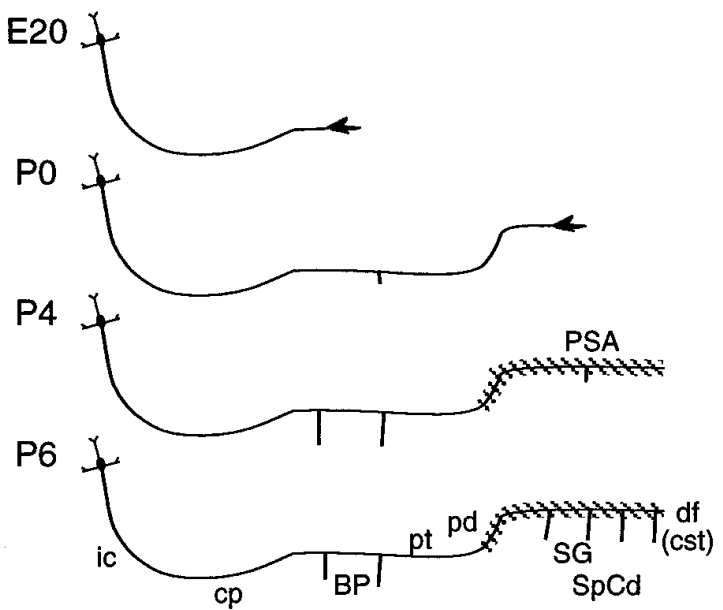

Figure 5. Developmental sequence of collateral branch formation and PSA expression on corticospinal axons. Corticospinal axons reach the basilar pons at E20 and form the first collateral branches to the pons at P0. In the spinal cord, collateral branch formation starts at P4. Whereas NCAM is present along the entire length of corticospinal axons at all ages, PSA is found only on the distal portion of these axons and only at later stages of their development. E20-P0, PSA is not detected on corticospinal axons as they grow from cortex to the spinal cord. P4-P6, PSA is upregulated on a restricted distal portion of corticospinal axons starting near the pyramidal decussation. The spatiotemporal dynamics of PSA localization on corticospinal axons correlates with their formation of collateral branches to the spinal gray. Refer to Figure $1 A$ for the anatomical context of these axons within the brain and spinal cord. Abbreviations: $B P$, basilar pons; $c p$, cerebral peduncle; $c s t$, corticospinal tract; $d f$, dorsal funiculus; $i c$, internal capsule; $L 5$, layer 5; $p d$, pyramidal decussation; $p t$, pyramidal tract; $S G$, spinal gray; $S p C d$, spinal cord.

\section{PSA removal affects corticospinal axon branching in the spinal cord}

The spatiotemporal distribution of PSA suggests that it might influence the innervation of the spinal gray by corticospinal axons. The following experiments were designed to test whether PSA may regulate the branching of corticospinal axons in the spinal cord by treating neonatal rats with endo $\mathrm{N}$. The endo $\mathrm{N}$ used in this study was purified free of any other enzymatic activity, resulting in an enzyme that specifically degrades PSA without otherwise altering NCAM or affecting any other sialic acid-containing structure (Vimr et al., 1984; Rustishauser et al., 1985; Hallenbeck et al., 1987).

\section{Endo $N$ eliminates PSA in the CST}

Rat pups were treated within hours of birth with endo N, heatinactivated endo $\mathrm{N}$, or sham injections in the fourth ventricle. Figure 6 shows examples of mAb 5A5 immunostaining of spinal cord sections from littermates perfused at P5. PSA staining appeared normal in the spinal cords of rat pups treated with heatinactivated endo $\mathrm{N}$ (Fig. $6 A$ ) or sham injections (not shown), with intense staining localized to the CST. In every rat pup injected with endo $\mathrm{N}$ (total $=21$ ), PSA staining was completely abolished in the spinal cord (Fig. 6C), whereas NCAM staining appeared normal (Fig. 6B,D). Identical results were seen when rat pups were sacrificed at P4 or P6 (not shown). These findings demonstrate that a single injection of endo $\mathrm{N}$ at P0 eliminates PSA throughout the spinal cord for at least the period of time covered in this study. 

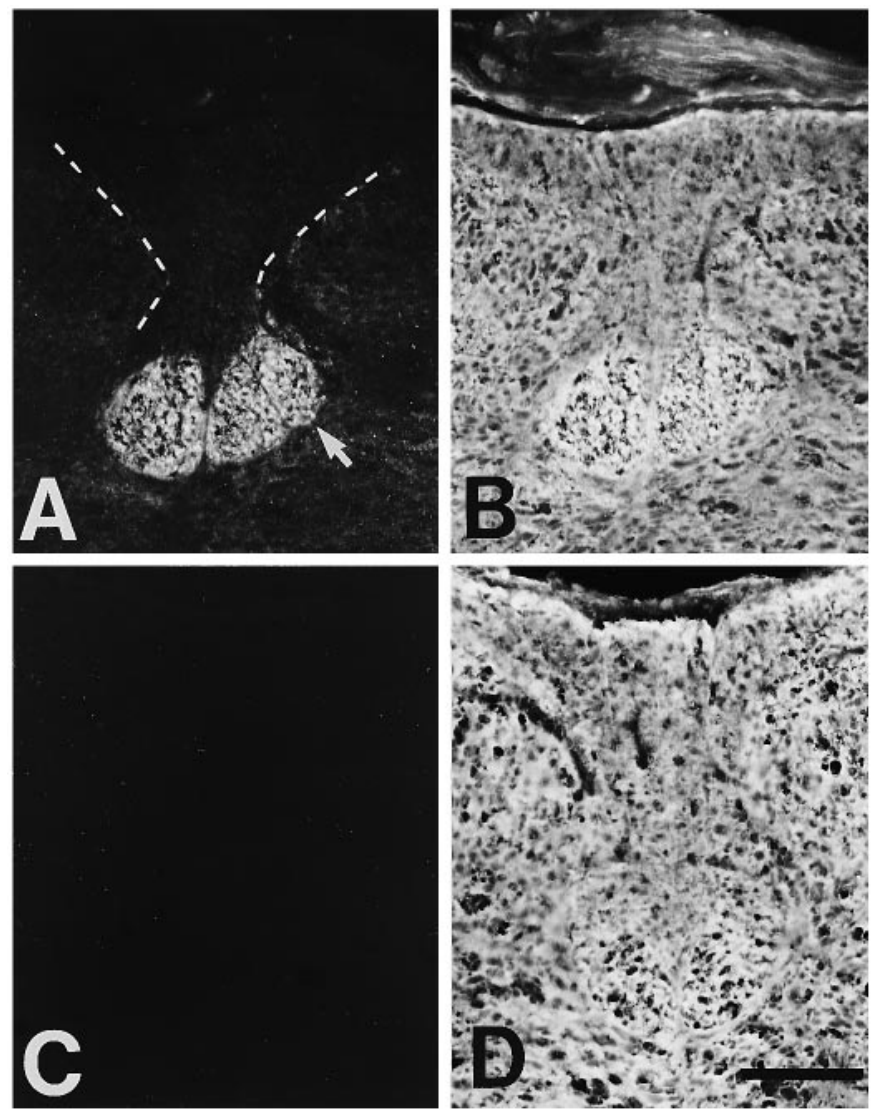

Figure 6. Endoneuraminidase $\mathrm{N}$ eliminates PSA in the CST. Transverse sections of P5 thoracic spinal cords, from rats treated at birth with heat-inactivated endo $\mathrm{N}(A, B)$ or active endo $\mathrm{N}(C, D)$, were doubleimmunostained for PSA $(A, C)$ and NCAM $(B, D)$. In control spinal cords, intense PSA staining is localized to the CST (arrow in $A$; the remainder of the dorsal funiculi are outlined by a dashed line and have much lower immunostaining for PSA), whereas intense NCAM staining is present throughout the spinal cord $(B)$. Endo $\mathrm{N}$ treatment eliminates PSA immunoreactivity $(C)$, but NCAM staining is unchanged $(D)$. Dorsal is to the top, lateral to the sides. The midline is at the middle of each panel. The approximate location and orientation of the panels is illustrated in Figure $1 B$ (shaded box). Scale bar (shown in $D$ ): $100 \mu \mathrm{m}$.

\section{Removal of PSA reduces collateral branching of corticospinal axons in the spinal cord}

During normal development of the rat corticospinal projection, corticospinal axons pass through the pyramidal decussation into the spinal cord on P0 and begin to form collaterals at cervical levels on $\mathrm{P} 4$. The collateral branches become more numerous during the following several days (Lopez-Mascaraque and O'Leary, 1991). We treated rat pups with endo $\mathrm{N}$, heat-inactivated endo $\mathrm{N}$, or sham injections on $\mathrm{P} 0$, and later labeled corticospinal axons with cortical injections of anterograde axonal tracers during the early and intermediate phases of collateral branching to determine whether PSA removal affected this process. The cervical portions of the spinal cords were used to assess axon branching. A segment of each spinal cord, taken from the mid-thoracic level, was used for anti-PSA immunostaining to verify the effectiveness of the endo $\mathrm{N}$ treatment (Fig. 6).

To assess qualitatively the effect of PSA removal, we injected DiI in sensorimotor cortex on P2 to label a large number of corticospinal axons. Rat pups were perfused at P4, P5, or P6. A total of 21 animals from three litters were used in this set of experiments (P4: four endo $\mathrm{N}$ and two heat-inactivated endo N; P5: four endo $\mathrm{N}$, two heat-inactivated endo $\mathrm{N}$, and two shaminjected; P6: five endo $\mathrm{N}$, one heat-inactivated endo $\mathrm{N}$, and one sham-injected). The corticospinal pathway in the endo N-treated rats appeared the same as that in normal littermates. We did not observe any unusual tendency for labeled axons to stray from the CST, nor other evidence for pathfinding errors (not shown). In contrast to this similarity in the trajectories of the parent corticospinal axons, we found a significant difference between endo $\mathrm{N}$-treated and normal rats in the onset and magnitude of corticospinal axon branching in the spinal cord. Figure 7 shows comparisons of axonal branching in the cervical region of spinal cords of control animals (left) and endo N-treated (right) littermates at P4, $\mathrm{P} 5$, and P6. At P4, collateral branches were clearly seen to extend laterally from the CST into the spinal gray in control animals; however, few or no branches were seen in endo N-treated littermates. In spinal cords examined at P5 and P6, collateral branches were present in endo N-treated animals but were more sparse compared to control littermates. These findings show that endo $\mathrm{N}$ treatment does not prevent the collateral branching of corticospinal axons, but instead attenuates branch formation as well as delays its onset.

DiI labeling of corticospinal axons resulted in a large number of labeled axons and was useful in gaining a qualitative impression of the extent of corticospinal axon branching. To facilitate quantification of the effect of endo $\mathrm{N}$ treatment on axon branching, biocytin was used to anterogradely label corticospinal axons. The lower density of labeled axons made it possible to count both axon numbers and individual branch points (Fig. $8 A$ ). Because at $\mathrm{P} 4$ we had found essentially an absolute difference in branching between endo N-treated and control animals, with no branching in the endo $\mathrm{N}$-treated cases compared to considerable branching in the control cases, we focused on quantifying axonal branching at P5, an age when collaterals were observed in both endo $\mathrm{N}$-treated and control spinal cords. Pups from two litters were treated at $\mathrm{P} 0$ with endo $\mathrm{N}(n=9)$ or used as controls (sham injections, $n=7$; heat-inactivated endo $\mathrm{N}, n=2$ ), labeled later with biocytin, and then analyzed at P5. The number of branches per labeled axon ranged from 0.30 to 1.38 with a mean of $0.72 \pm 0.11$ SEM in endo $\mathrm{N}$-treated animals (375 axons analyzed), and from 0.42 to 1.98 with a mean of $1.09 \pm 0.16 \mathrm{SEM}$ in control animals (426 axons analyzed). Thus, endo $\mathrm{N}$ treatment resulted in a statistically significant reduction of $34 \%$ in the mean number of branches/axon (Student's $t$ test; $t=1.88 ; \mathrm{df}=16 ; p<0.05 ;$ Fig. 7B). Because in every case the endo $\mathrm{N}$ treatment removed all immunohistochemically detectable PSA from the spinal cord, the overlap between these two populations is likely attributable to the inherent developmental variability normally observed among individuals rather than differences in the effectiveness of the endo $\mathrm{N}$ treatment. These results indicate that the reduction in corticospinal axon branching in the spinal cord after endo $\mathrm{N}$ treatment is specific and attributable to the selective removal of PSA.

\section{DISCUSSION}

By examining the normal pattern of PSA distribution in the developing corticospinal pathway and by assessing the effects of enzymatically removing PSA in vivo, we have provided evidence that PSA regulates the collateral branching of corticospinal axons. PSA is not present initially on growing corticospinal axons, but is later selectively upregulated only on the spinal segment of these axons at the time they begin to form collateral branches to the 


\section{Control}
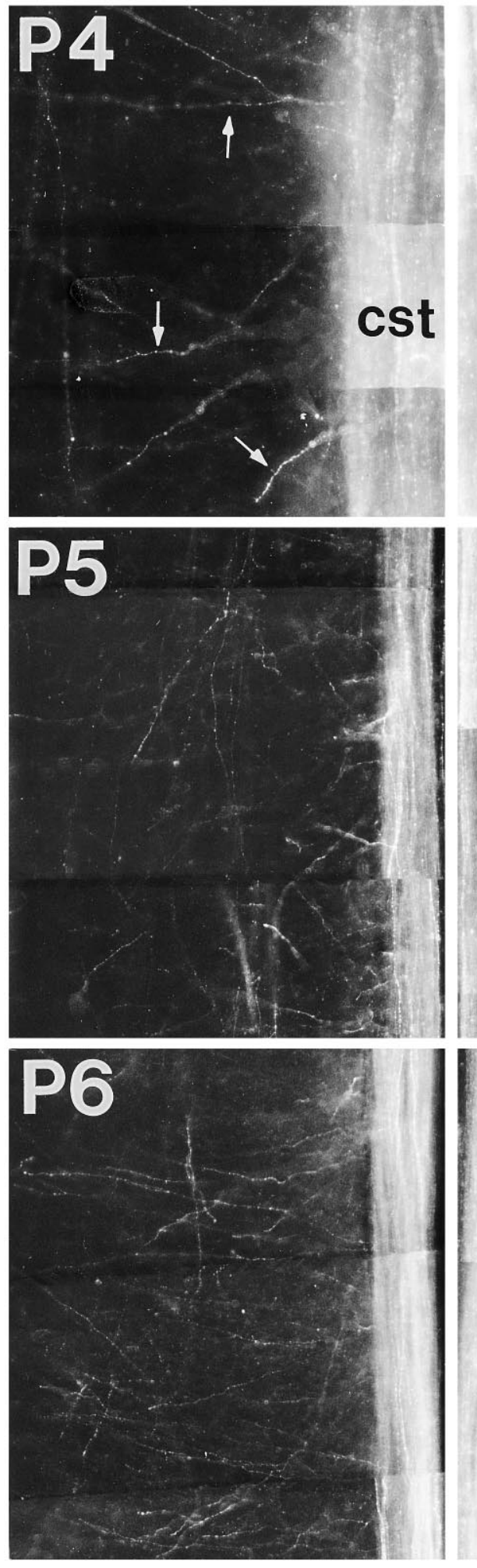

\section{Endo $\mathbf{N}$}
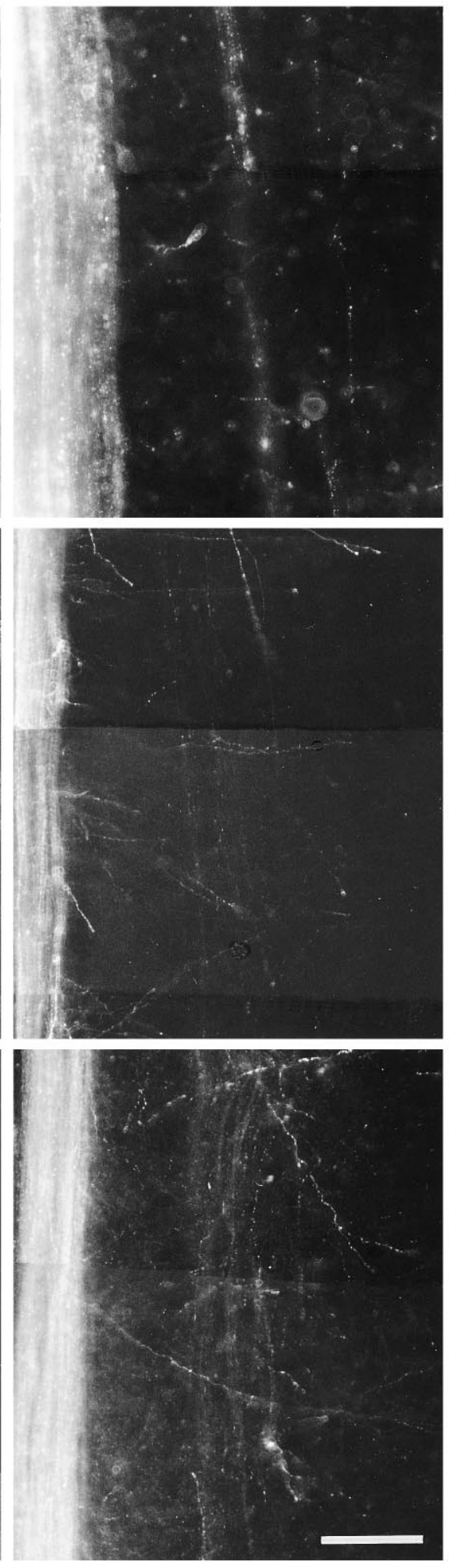

Figure 7. Removal of PSA delays and reduces collateral branching along corticospinal axons in the spinal cord. Horizontal sections (100 $\mu \mathrm{m}$ thick) through the CST of cervical spinal cords showing DiI-labeled corticospinal axons in control rats (left side) and endo $\mathrm{N}$-treated littermates (right side) at P4, P5, and P6. Spinal cords with similar densities of labeled corticospinal axons were chosen for comparison. Sections were matched on the basis of their dorsoventral position within the spinal cord. At P4, in control animals collateral branches (arrows) extend laterally from the CST into the spinal gray; however, few or no branches were seen in endo N-treated littermates. At P5 and P6, collateral branches were present in endo N-treated animals but were more sparse compared with control littermates. Each panel shows one side of the spinal cord; the CST is at the midline of the spinal cord, and the branches extend laterally from it into the spinal gray. Rostral is to the top. The approximate location and orientation of these panels are illustrated in Figure 1, $A$ (arrow marked $H$ ) and $C$. Scale bar, $200 \mu \mathrm{m}$. spinal gray. The selective removal of PSA delays the onset of collateral branching by corticospinal axons within the spinal cord and later diminishes the magnitude of their branching. These findings are consistent with the notion that PSA modifies axonal responses to external stimuli rather than directly stimulating axonal responses.

We chose to use an enzymatic removal of PSA with endo $\mathrm{N}$ for this study because it completely abolishes PSA for extended 

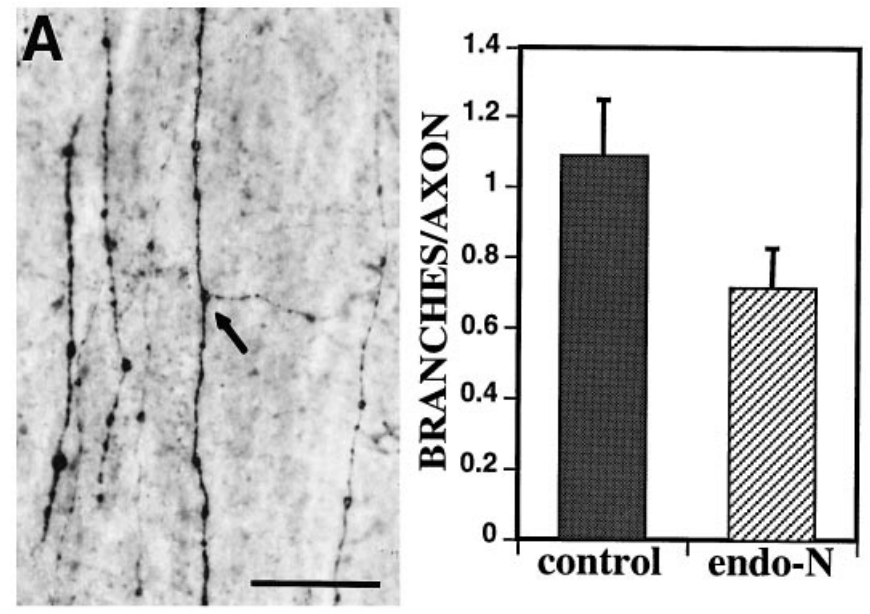

Figure 8. Quantification of the effect of PSA removal on corticospinal axon branching in the spinal cord. Because few or no branches were evident in the P4 endo N-treated cases, we focused on quantifying axonal branching at P5, an age when collateral branches were observed in both endo N-treated and control spinal cords. $A$, Horizontal sections $(50 \mu \mathrm{m}$ thick) through the CST of P5 cervical spinal cord showing biocytin-labeled corticospinal axons. The lower density of axons labeled with biocytin compared to DiI made it possible to discern and count both axons and branch points (arrow). Scale bar, $50 \mu \mathrm{m}$. B, Endo N treatment resulted in a statistically significant $34 \%$ reduction in the average number of branches/axon normally present at P5. Bars indicate SEM.

periods of time, leaves the NCAM polypeptide intact, and is nontoxic (Hallenbeck et al., 1987; Rutishauser, 1989). An alternative approach would have been to use a knockout mouse lacking all isoforms of NCAM (Cremer et al., 1994) or specifically NCAM 180 (Tomasiewicz et al., 1993). The predominant reported anatomical defect in these mice, an abnormally small olfactory bulb that results from an aberrant migration of precursor cells from the telencephalic neuroepithelium, is apparently attributable to the lack of PSA, because the knockout mice lack PSA in the olfactory bulb and the mutant phenotype can be mimicked by enzymatic removal of PSA (Ono et al., 1994). A priori, however, endo $\mathrm{N}$ treatment is a more straightforward strategy for selectively studying the functions of PSA, because it clearly dissociates the effect of PSA removal from the effect of NCAM removal.

Heterogeneity in the distribution of PSA-modified NCAM on individual cells has been shown previously for retinal ganglion cells, which have PSA along the entire length of their axons but not on their cell bodies (Schlosshauer et al., 1984). An important feature of PSA expression in the corticospinal pathway is its differential distribution along corticospinal axons. Similarly, some axonal glycoproteins, such as TAG-1 and L1 on rat spinal cord commissural axons (Dodd et al., 1988) and fasciclins on insect neurons (Bastiani et al., 1987; Patel et al., 1987), have been localized to specific axon segments. PSA is distinct from these examples, however, in that it involves the discontinuous distribution of a post-translational modification and not necessarily the polypeptide. The localization of PSA to one portion of corticospinal axons could be achieved in at least two ways. The PSA modification could be restricted to an NCAM isoform that is not uniformly distributed. Indeed, both major transmembrane forms of NCAM (NCAM-140 and NCAM-180) can assume a polarized or nonuniform distribution on cells (Persohn et al., 1989; Gunderson et al., 1993), and the specific targeting of PSA to distinct regions of myotube cell surfaces reflects the differential distribu- tion of the lipid-linked form of NCAM (i.e., NCAM-120) to which the PSA is attached (Fredette et al., 1993). In the olfactory bulb, PSA is associated with NCAM 180 (Ono et al., 1994), but it is not known which NCAM isoforms are involved in the upregulation of PSA in the CST. Alternatively, the PSA modification of a homogeneously distributed NCAM isoform could be restricted to the distal portion of an axon by either directed targeting of the PSA-modified NCAM or by local differences in the modification of NCAM after integration into the axonal membrane.

The developmental events leading to target innervation by corticospinal axons seems to be similar in the brainstem and spinal cord (O'Leary et al., 1990). In both cases, corticospinal axons extend out of the cortex and along their subcortical pathway, bypassing their brainstem and spinal targets. Subsequently, these targets are contacted by collaterals that branch off of the axon shaft well behind the advancing growth cone (O'Leary and Terashima, 1988; O'Leary et al., 1990; Kuang and Kalil, 1994; Bastmeyer and O'Leary, 1996). Even though the process of target innervation by corticospinal axons is similar within the brainstem and spinal cord, axon collateralization at these distinct sites seems to be regulated differentially, given the dramatic difference in PSA content on NCAM distributed along corticospinal axons. As an optimizer of responses to environmental cues, PSA may be required only in situations in which axon fasciculation is strong relative to external cues. Consistent with this suggestion, the cross-sectional area of the CST is much greater overlying the basilar pons than in the spinal cord, whereas axonal number is similar at these two locations during development (M. M. Daston, M. Bastmeyer, and D. D. M. O'Leary, unpublished observations). Thus, the packing density of corticospinal axons is much greater, and therefore fasciculation is probably tighter in the spinal cord than in the pons. Another possible explanation is that it may be more critical to generate branches at an accelerated schedule and higher number in the spinal cord than in the pons. This could be because the basilar pons is a relay station between cortex and the cerebellum, whereas the corticospinal innervation more directly controls motor output.

The chemical characteristics of PSA provide clues as to how it may function in neural development. Estimates of the size of the PSA moiety on highly sialated membranes suggest that the volume of PSA is several times that of the NCAM polypeptide (Rutishauser et al., 1988). In vitro, the removal of PSA from NCAM increases the rate of aggregation of membrane vesicles (Hoffman and Edelman, 1983; Rutishauser et al., 1985) and results in a fivefold increase in the area of close membrane apposition between cells (Rutishauser et al., 1988). Analysis of the physical properties of PSA on NCAM and at the cell surface (Yang et al., 1992, 1994) suggests that attenuation of cell-cell interactions stems not only from the steric properties of this carbohydrate, but also from its negative charge. In the chick PNS, the inhibition of axonal fasciculation by PSA on NCAM can be accounted for by the disruption of homophilic L1 binding. It has been suggested that this disruption of L1 binding is attributable to the sizable length of the PSA-modified NCAM moiety relative to the length of the extracellular domain of L1, thereby diminishing homophilic L1 interactions between neighboring axons (Landmesser et al., 1990). L1 is on corticospinal axons (Joosten and Gribnau, 1989) and could thus be involved in PSA effects in this system as well.

During the period of axon branching, CST axons in the spinal cord are surrounded almost entirely by other unmyelinated axons (Gorgels et al., 1989; Joosten et al., 1989). Thus, in this system 
PSA removal must exert its effects by altering relationships between neighboring axons. The ability of PSA to modulate axonaxon interactions has been demonstrated by both in vitro and in vivo experiments. For example, PSA removal enhances the fasciculation of neurites extended by cultured DRG neurons (Rutishauser et al., 1985). Removal of PSA from motor axons during their innervation of chick hindlimb muscles causes not only an increase in axon-axon bundling among fast motor fibers, but also a decrease in axon sprouting among slow motor fibers (Landmesser et al., 1990). It seems then that axon-axon fasciculation or the "escape" of a growth cone in forming a sprout is particularly susceptible to the removal of PSA.

PSA removal may exert its effect on corticospinal axon branching in one of several ways. One possibility is that stronger interactions and tighter axon fasciculation among CST axons attributable to PSA removal may interfere mechanically with branch formation. Alternatively, PSA removal may alter axonal responses to molecules that positively or negatively regulate branching. For example, PSA removal could bring axons into closer proximity, allowing for interactions with ligands on adjacent corticospinal axons or on other cell types within the CST, that inhibit branch formation. Another possibility is that PSA removal might reduce interactions between axons and branch-promoting molecules. In vitro studies suggest that the basilar pons releases a soluble chemoattractant that can induce branching and directed growth of corticospinal axons (Heffner et al., 1990; O'Leary et al., 1991; Sato et al., 1994). The same or similar activity may be released by the spinal gray (Joosten et al., 1991, 1994). The neurotrophin NT-3 is another potential branching cue, as it has been shown to induce premature "sprouting" of corticospinal axons when introduced into the spinal cords of early postnatal rats (Schnell et al., 1994) and can exert a tropic effect on cortical axons in vitro (O'Leary and Daston, 1994). If a soluble target-derived branching agent operates in CST development, PSA removal may diminish axon branching by causing tighter axon fasciculation and restricting diffusion of the branching agent into the CST. In any case, the normal role of PSA may be to attenuate axonal interactions within the CST and thereby allow branching cues to exert a greater influence on the axon shaft.

\section{REFERENCES}

Acheson A, Sunshine JL, Rutishauser U (1991) NCAM polysialic acid can regulate both cell-cell and cell-substrate interactions. J Cell Biol 114:143-153.

Adams JC (1992) Biotin amplification of biotin and horseradish peroxidase signals in histochemical stains. J Histochem Cytochem 40:1457-1463.

Bastiani MJ, Harrelson AL, Snow PM, Goodman CS (1987) Expression of fasciclin 1 and 11 glycoproteins on subsets of axon pathways during neuronal development in the grasshopper. Cell 48:745-755.

Bastmeyer M, O'Leary DDM (1996) Dynamics of target recognition by interstitial axon branching along developing cortical axons. J Neurosci 16:1450-1459.

Bhide PG, Frost DO (1991) Stages of growth of hamster retinofugal axons: implications for developing axonal pathways with multiple targets. J Neurosci 11:485-504.

Chuong C-M, Edelman GM (1984) Alterations in neural cell adhesion molecules during development of different regions of the nervous system. J Neurosci 4:2354-2368.

Cremer H, Lange R, Christoph A, Plomann M, Vopper G, Roes J, Brown R, Baldwin S, Kraemer P, Scheff S, Bathels D, Rajewsky K, Wille W (1994) Inactivation of the N-CAM gene in mice results in size reduction of the olfactory bulb and deficits in spatial learning. Nature 367:455-459.

Dodd J, Morton SB, Karagogeos D, Yamamoto M, Jessell TM (1988) Spatial regulation of axonal glycoprotein expression on subsets of embryonic spinal neurons. Neuron 1:105-116.
Fredette B, Rutishauser U, Landmesser L (1993) Regulation and activity-dependence of N-cadherin, NCAM isoforms and polysialic acid on chick myotubes during development. J Cell Biol 123:1867-1888.

Gorgels TGMF, DeKort EJM, Van Aanholt HTH, Nieuwenhuys R (1989) A quantitative analysis of the development of the pyramidal tract in the cervical spinal cord in the rat. Anat Embryol 179:377-385.

Gunderson D, Powell SK, Rodriquez-Boulan E (1993) Apical polarization of NCAM in retinal pigment epithelium is dependent on contact with the neural retina. J Cell Biol 121:335-343.

Hallenbeck PC, Vimr ER, Yu F, Bassier B, Troy FA (1987) Purification and properties of a bacteriophage-induced endo- $N$-acetylneuraminidase specific for poly-alpha-2,8-sialosyl carbohydrate units. J Biol Chem 262:3553-3561.

Heffner CD, Lumsden AGS, O'Leary DDM (1990) Target control of collateral extension and directional axon growth in the mammalian brain. Science 247:217-220.

Hoffman S, Edelman GM (1983) Kinetics of homophilic binding by embryonic and adult forms of the neural cell adhesion molecule. Proc Natl Acad Sci USA 80:5762-5766.

Hoffman S, Sorkin BC, White PC, Brackenbury R, Mailhammer R, Rutishauser U, Cunningham BA, Edelman GM (1982) Chemical characterization of a neural cell adhesion molecule (NCAM) purified from embryonic brain membranes. J Biol Chem 257:7720-7729.

Honig MG, Hume RI (1989) DiI and DiO: versatile fluorescent dyes for neuronal labeling and pathway tracing. Trends Neurosci 12:333-341.

Joosten EAJ (1994) Developmental expression of N-CAM epitopes in the rat spinal cord during corticospinal tract axon outgrowth and target innervation. Dev Brain Res 78:226-236.

Joosten EAJ, Gribnau AAM (1989) Immunocytochemical localization of cell adhesion molecule L1 in developing rat pyramidal tract. Neurosci Lett 100:94-98.

Joosten EAJ, Gribnau AAM, Dederen PJWC (1989) Postnatal development of the corticospinal tract in the rat: an ultrastructural anterograde HRP study. Anat Embryol 179:449-456.

Joosten EAJ, van der Ven PFM, Hooiveld MHW, ten Donkelaar HJ (1991) Induction of corticospinal target finding by release of a diffusible, chemotropic factor in cervical spinal grey matter. Neurosci Lett 128:25-28.

Joosten EAJ, Gispen WH, Bar PR (1994) Tropism and corticospinal target selection in the rat. Neuroscience 59:33-41.

Kuang RZ, Kalil K (1994) Development of specificity in corticospinal connections by collaterals branching selectively into appropriate spinal targets. J Comp Neurol 344:270-282.

Landmesser L, Dahm L, Tang J, Rutishauser U (1990) Polysialic acid as a regulator of intramuscular nerve branching during embryonic development. Neuron 4:655-667.

Liu W-L, Behbehani MM, Shipley MT (1993) Intracellular filling in fixed brain slices using Miniruby, a fluorescent biocytin compound. Brain Res 608:78-86.

Lopez-Mascaraque L, O'Leary DDM (1991) Specificity and mode of growth of developing corticospinal axons to their spinal gray terminal fields. Soc Neurosci Abstr 17:740.

Nakamura H, O'Leary DDM (1989) Inaccuracies in initial growth and arborization of chick retinotectal axons followed by course corrections and axon remodeling to develop topographic order. J Neurosci 9:3776-3795.

O'Leary DDM, Daston MM (1994) Neurotrophin-3 has a chemotropic effect on cortical neurons. Soc Neurosci Abstr 20:1685a.

O'Leary DDM, Koester SE (1993) Development of projection neuron types, axon pathways, and patterned connections of the mammalian cortex. Neuron 10:991-1006.

O'Leary DDM, Terashima T (1988) Cortical axons branch to multiple subcortical targets by interstitial axon budding: implications for target recognition and "waiting periods." Neuron 1:901-910.

O'Leary DDM, Bicknese AR, De Carlos JA, Heffner CD, Koester SE, Kutka LJ, Terashima T (1990) Target selection by cortical axons: alternative mechanisms to establish axonal connections in the developing brain. In: Cold Spring Harbor Symposia on Quantitative Biology, vol LV, pp 453-367. Cold Spring Harbor, New York: Cold Spring Harbor Laboratory.

O'Leary DDM, Heffner CD, Kutka L, Lopez-Mascaraque L, Missias A, Reinoso BS (1991) A target-derived chemoattractant controls the development of the corticopontine projection by a novel mechanism of axon targeting. Development [Suppl] 2:123-130. 
Ono K, Tomasiewicz H, Magnuson T, Rutishauser U (1994) N-CAM mutation inhibits tangential neuronal migration and is phenocopied by enzymatic removal of polysialic acid. Neuron 13:595-609.

Patel NH, Snow PM, Goodman CS (1987) Characterization and cloning of fasciclin III: a glycoprotein expressed on a subset of neurons and axon pathways in Drosophila. Cell 48:975-988.

Persohn E, Pollerberg G E, Schachner M (1989) Immunoelectronmicroscopic localization of the $180 \mathrm{kDa}$ component of the neural cell adhesion molecule NCAM in postsynaptic membranes. J Comp Neurol 288:92-100.

Rothbard JB, Brackenbury R, Cunningham BA, Edelman GM (1982) Differences in the carbohydrate structures of neural cell adhesion molecules from adult and embryonic chicken brains. J Biol Chem 257:11064-11069.

Rougon G, Deagostini-Bazin H, Hirn M, Giordis C (1982) Tissue and developmental stage-specific forms of a neural cell-surface antigen linked to differences in glycosylation of a common polypeptide. EMBO J 1:1239-1244.

Rutishauser U (1989) Polysialic acid as a regulator of cell interactions. In: Neurobiology of glycoconjugates (Margolis RU, Margolis RK, eds), pp 367-382. New York: Plenum.

Rutishauser U, Watanabe M, Silver J, Troy FA, Vimr ER (1985) Specific alteration of NCAM-mediated cell adhesion by an endoneuraminidase. J Cell Biol 101:1842-1849.

Rutishauser U, Acheson A, Hall AK, Mann DM, Sunshine J (1988) The neural cell adhesion molecule (NCAM) as a regulator of cell-cell interactions. Science 240:53-57.

Sato M, Lopez-Mascaraque L, Heffner CD, O'Leary DDM (1994) Action of a diffusible target-derived chemoattractant on cortical axon branch induction and directed growth. Neuron 13:791-803.

Schlosshauer B, Schwarz U, Rutishauser U (1984) Topological distribution of different forms of neural cell adhesion molecule in the developing chick visual system. Nature 310:141-143.
Schnell L, Schneider R, Kolbeck R, Barde YA, Schwab MD (1994) Neurotrophin-3 enhances sprouting of corticospinal tract axons during development and after adult spinal cord lesion. Nature 367:170-173.

Schreyer DJ, Jones EG (1982) Growth and target finding by axons of the corticospinal tract in prenatal and postnatal rats. Neuroscience 7:1837-1853.

Snider WD, Zhang L, Yusoof S, Gorukanti N, Tsering C (1992) Interactions between dorsal root axons and their target motor neurons in developing mammalian spinal cord. J Neurosci 12:3494-3508.

Stanfield BB (1992) The development of the corticospinal projection. Prog Neurobiol 38:169-202.

Stanfield BB, Nahin BR, O'Leary DDM (1987) A transient postmamillary component of the rat fornix during development: implications for interspecific differences in mature axonal projections. J Neurosci 7:3350-3361.

Tang J, Landmesser L, Rutishauser U (1992) Polysialic acid influences specific pathfinding by avian motoneurons. Neuron 8:1031-1044.

Tang J, Rutishauser U, Landmesser L (1994) Polysialic acid regulates growth cone behavior during sorting of motor axons in the plexus region. Neuron 13:405-414.

Tomasiewicz H, Ono K, Yee D, Thompson C, Giordis C, Rutishauser U, Magnuson T (1993) Genetic deletion of a neural cell adhesion molecule variant (N-CAM-180) produces distinct defects in the central nervous system. Neuron 11:1163-1174.

Vimr ER, McCoy RD, Vollger HF, Wilkinson NC, Troy FA (1984) Use of prokaryotic-derived probes to identify polysialic acid in neonatal membranes. Proc Natl Acad Sci USA 81:1971-1975.

Yang P, Yin X, Rutishauser U (1992) Intercellular space is affected by the polysialic acid content of NCAM. J Cell Biol 116:1487-1496.

Yang P, Major D, Rutishauser U (1994) Role of charge and hydration in effects of polysialic acid on molecular interactions on and between cell membranes. J Biol Chem 269:23039-23044. 